\title{
Anisotropic lattice QCD study of pentaquark baryons in spin $3 / 2$ channel
}

Takumi Doi* ${ }^{\dagger}$

RIKEN BNL Research Center, Brookhaven National Laboratory, Upton, New York 11973, USA

E-mail: doi@quark.phy.bnl.gov

\section{Noriyoshi Ishii}

Department of Physics, H-27, Tokyo Institute of Technology,

2-12-1 Oh-okayama, Meguro, Tokyo 152-8551, Japan

E-mail: ishii@rarfaxp.riken.jp

Yukio Nemoto

Department of Physics, Nagoya University, Furo, Chikusa, Nagoya 464-8602, Japan

E-mail: nemoto@hken.phys.nagoya-u.ac.jp

\section{Makoto Oka}

Department of Physics, H-27, Tokyo Institute of Technology,

2-12-1 Oh-okayama, Meguro, Tokyo 152-8551, Japan

E-mail: oka@th.phys.titech.ac.jp

\section{Hideo Suganuma}

Department of Physics, Kyoto University, Kitashirakawaoiwake, Kyoto 606-8502, Japan

E-mail: suganuma@th.phys.titech.ac.jp

We perform the comprehensive analysis of the pentaquark (5Q) in $J^{P}=3 / 2^{ \pm}$channel using anisotropic quenched lattice QCD. We employ the standard Wilson gauge action at $\beta=5.75$ and the $O(a)$ improved Wilson (clover) quark action on a $12^{3} \times 96$ lattice with the renormalized anisotropy as $a_{\mathrm{s}} / a_{\mathrm{t}}=4$. A large number of gauge configurations as $N_{\text {conf }}=1000$ is analyzed, which is found to be essential to achieve a reliable measurement. We study three types of the Rarita-Schwinger intepolationg fields with $I=0$ : (a) the $\mathrm{NK}^{*}$-type, (b) the (color-)twisted NK*type, (c) a diquark-type. As a result, we find only massive states as $m_{5 \mathrm{Q}} \simeq 2.1-2.2 \mathrm{GeV}$ in $J^{P}=3 / 2^{-}$channel, and $m_{5 \mathrm{Q}}=2.4-2.6 \mathrm{GeV}$ in $J^{P}=3 / 2^{+}$channel in the chiral limit. The analysis with the hybrid boundary condition (HBC) is performed to distinguish whether these resonances are compact 5Q resonances or two-particle scattering states. No low-lying compact $5 \mathrm{Q}$ resonance states are found below $2.1 \mathrm{GeV}$.

XXIIIrd International Symposium on Lattice Field Theory

25-30 July 2005

Trinity College, Dublin, Ireland

\footnotetext{
*Speaker.

${ }^{\dagger}$ The Monte Carlo simulations have been performed on the NEC SX-5 supercomputer at Osaka University.
} 


\section{Introduction}

The announcement of the discovery of the new-particle $\Theta^{+}$(1540) with narrow width by the LEPS group at SPring-8[1] has triggered enormous studies to understand this mysterious state. In fact, $\Theta^{+}$has baryon number $B=+1$, charge $Q=+1$ and strangeness $S=+1$, and therefore has the minimal configuration of $u u d d \bar{s}$, which is manifestly exotic. Although the impact of this discovery is undoubted, the experimental status is quite controversial: several groups confirmed the existence of $\Theta^{+}[2]$, while others reported the null results[2]. Note that even positive results have not determined the quantum numbers such as spin and parity. Numerous theoretical studies have been performed as well to understand the nature of $\Theta^{+}$[3]. In particular, one of the central issues is to realize the mechanism for the extremely narrow width as $\Gamma \lesssim 1 \mathrm{MeV}$. Among several scenarios proposed so far, $J^{P}=3 / 2^{-}$possibility[4] is interesting to investigate. In fact, if $\Theta^{+}$is $J^{P}=3 / 2^{-}$ state, the decay to the KN scattering state becomes the d-wave. Because it is expected that the special configuration $(0 s)^{5}$ is dominant in the ground-state in $J^{P}=3 / 2^{-}$channel, the decay to the dwave is suppressed by the wave function factor. Note that there is further suppression by the d-wave centrifugal barrier, leading to the significantly narrow decay width. However, this mechanism has a possible disadvantage that such a state tends to be massive due to the color-magnetic interaction in the constituent quark models. $J^{P}=3 / 2^{+}$possibility attracts another interest considering the diquark model[5]. In this model, $J^{P}=1 / 2^{+}$is predicted for $\Theta^{+}$, where $\Theta^{+}$is composed of an anti s quark and two scalar diquarks with relative p-wave angular momentum. Narrow width is expected from this exotic structure as well as the p-wave centrifugal barrier. If QCD really exhibits this scenario, we expect that $J^{P}=3 / 2^{+}$state also exists as a LS-partner of $\Theta^{+}$. Under these circumstances, we present anisotropic lattice QCD results on 5Q states in $J^{P}=3 / 2^{ \pm}$channels. Note that while there have been several lattice QCD calculations of $5 \mathrm{Q}$ states $[6,7,8,9,10,11,12$, $13,14,15,16]$, these studies are restricted to $J^{P}=1 / 2^{ \pm}$channels except for a very recent one [16].

\section{Formalism}

We study the pentaquark (5Q) state analyzing the Euclidean two-point correlator as $G_{\mu \nu}(\tau) \equiv$ $\sum_{\vec{x}}\left\langle\psi_{\mu}(\tau, \vec{x}) \bar{\psi}_{v}(0, \overrightarrow{0})\right\rangle$, where $\psi_{\mu}$ is a Rarita-Schwinger interpolating field. In principle, we can use any field $\psi_{\mu}$ with appropriate quantum numbers. In practice, however, some fields may couple strongly to a genuine 5Q state, while other fields may couple weakly. Unfortunately, there is no empirical knowledge about the structure of the 5Q state, i.e., about the suitable interpolating field, we perform the comprehensive study using the three types of fields and examine how the results depend on the choice of the fields. We employ following isoscalar fields: (a) NK*type, $\psi_{\mu} \equiv \varepsilon_{a b c}\left(u_{a}^{T} C \gamma_{5} d_{b}\right) u_{c} \cdot\left(\bar{s}_{d} \gamma_{\mu} d_{d}\right)+(u \leftrightarrow d)(\mathrm{b})$ (color-)twisted NK*-type, which is an extention to the one in Ref.[6], $\psi_{\mu} \equiv \varepsilon_{a b c}\left(u_{a}^{T} C \gamma_{5} d_{b}\right) u_{d} \cdot\left(\bar{s}_{d} \gamma_{\mu} d_{c}\right)+(u \leftrightarrow d)$ (c) diquark-type[7], which is an extention to the one in Ref.[17], $\psi_{\mu} \equiv \varepsilon_{a b c} \varepsilon_{d e f} \varepsilon_{c f g}\left(u_{a}^{T} C \gamma_{5} d_{b}\right)\left(u_{d}^{T} C \gamma_{5} \gamma_{\mu} d_{e}\right) C \gamma_{5} \bar{s}_{g}$. To analyze spin $3 / 2$ state, we decompose the correlator as $G_{i j}(\tau)=\mathbf{P}_{i j}^{(3 / 2)} G^{(3 / 2)}(\tau)+\mathbf{P}_{i j}^{(1 / 2)} G^{(1 / 2)}(\tau)$, where $G^{(3 / 2)}(\tau)$ and $G^{(1 / 2)}(\tau)$ denote the spin $3 / 2$ and $1 / 2$ contributions to $G(\tau)$, respectively, and $\mathbf{P}_{i j}^{(3 / 2)} \equiv \delta_{i j}-(1 / 3) \gamma_{i} \gamma_{j}, \mathbf{P}_{i j}^{(1 / 2)} \equiv(1 / 3) \gamma_{i} \gamma_{j}$. In order to study both parity states, the parity projection is performed as well with $P_{ \pm} \equiv\left(1 \mp \gamma_{4}\right) / 2$. Note that all of the fields transform as $\psi_{i}(\tau, \vec{x}) \rightarrow$ $-\gamma_{4} \psi_{i}(\tau,-\vec{x})(i=1,2,3)$ under the spatial reflection of quark fields as $q(\tau, \vec{x}) \rightarrow+\gamma_{4} q(\tau,-\vec{x})$. 
In the 5Q state study from lattice $\mathrm{QCD}$, one of the most important issues is the discrimination between two-particle scattering states and genuine 5Q compact resonances. In fact, in $J=3 / 2, I=0$ channel, $\mathrm{NK}$ and $\mathrm{NK}^{*}$ scattering states are expected to have large contribution in the $5 \mathrm{Q}$ correlator. To resolve this problem, we use two distinct spatial boundary conditions(BC), i.e., the periodic $\mathrm{BC}(\mathrm{PBC})$ and the hybrid $\mathrm{BC}(\mathrm{HBC})$, which is recently proposed in Ref.[10]. In PBC, we impose the spatially periodic $\mathrm{BC}$ on $\mathrm{u}, \mathrm{d}$ and s-quarks. As a result, all the hadrons are subject to the periodic $\mathrm{BC}$, and their momenta are quantized as $p_{i}=2 n_{i} \pi / L,\left(n_{i} \in \mathbb{Z}\right)$, where $L$ denotes the spatial extent of the lattice. On the other hand, in $\mathrm{HBC}$, we impose the spatially anti-periodic BC on $\mathrm{u}$ and d-quarks, whereas the spatially periodic BC is imposed on s-quark. Since $\mathrm{N}(u u d, u d d)$, $\mathrm{K}(u \bar{s}, d \bar{s})$ and $\mathrm{K}^{*}(u \bar{s}, d \bar{s})$ contain odd numbers of $\mathrm{u}$ and $\mathrm{d}$ quarks, they are subject to the anti-periodic $\mathrm{BC}$ and the momenta are quantized as $p_{i}=\left(2 n_{i}+1\right) \pi / L$. The crucial point is that the energy of the low-lying two-particle scattering state, $E_{\min } \simeq \sqrt{m_{N}^{2}+\left|\vec{p}_{\min }\right|^{2}}+\sqrt{m_{\tilde{K}}^{2}+\left|\vec{p}_{\min }\right|^{2}}\left(\tilde{K}=K\right.$ or $\left.K^{*}\right)$, is different between PBC and HBC because of the difference of the quantization of momenta. In fact, a drastic change is expected in the s-wave $\mathrm{NK}^{*}$ state. In PBC, the lowest energy of $\mathrm{NK}^{*}$ state is $E_{\min }^{\mathrm{PBC}} \simeq m_{N}+m_{K^{*}}$. In contrast, since both $\mathrm{N}$ and $\mathrm{K}^{*}$ are required to have non-vanishing momenta $( \pm \pi / L, \pm \pi / L, \pm \pi / L)$ in $\mathrm{HBC}$, the lowest energy is raised up as $E_{\min }^{\mathrm{HBC}} \simeq \sqrt{m_{N}^{2}+3 \pi^{2} / L^{2}}+$ $\sqrt{m_{K^{*}}^{2}+3 \pi^{2} / L^{2}}$, where the shift of energy amounts typically to a few hundred $\mathrm{MeV}$ for $L \sim 2$ $\mathrm{fm}$. The energy shift appears in NK(d-wave), NK(p-wave), $\mathrm{NK}^{*}$ (p-wave) as well. Note that the minimum momentum in PBC is not $\vec{p}=\overrightarrow{0}$, but $\vec{p}=( \pm 2 \pi / L, 0,0),(0, \pm 2 \pi / L, 0),(0,0, \pm 2 \pi / L)$, because $\vec{p}=\overrightarrow{0}$ state can couple only to s-wave scattering states. Therefore, the lowest energies are expressed as $E_{\min }^{\mathrm{PBC}} \simeq \sqrt{m_{N}^{2}+4 \pi^{2} / L^{2}}+\sqrt{m_{\tilde{K}}^{2}+4 \pi^{2} / L^{2}}$ and $E_{\min }^{\mathrm{HBC}} \simeq \sqrt{m_{N}^{2}+3 \pi^{2} / L^{2}}+$ $\sqrt{m_{\tilde{K}}^{2}+3 \pi^{2} / L^{2}}$. These energy difference between PBC and HBC of scattering states contrast strongly with one in a compact $5 \mathrm{Q}$ resonance. In fact, because $\Theta^{+}(u u d d \bar{s})$ contains even number of $\mathrm{u}$ and d quarks, it is subject to the spatially periodic BC not only in PBC but also in HBC, which allows $\Theta^{+}$to have $\vec{p}=\overrightarrow{0}$ in both of PBC and HBC. Therefore, the energy difference of a compact 5Q state is expected to be marginal, since it originates only from the change in its intrinsic structure. In this way, we can identify whether the observed states on the lattice are compact 5Q resonances or scattering states, by examining the energy difference between PBC and HBC.

\section{Lattice QCD parameters and Numerical results}

We calculate 5Q correlators using anisotropic quenched lattice QCD. To generate gauge field configurations, we adopt the standard Wilson gauge action at $\beta=5.75$ on the $12^{3} \times 96$ lattice with the renormalized anisotropy $a_{s} / a_{t}=4$. Note that the anisotropic lattice is suitable for highprecision measurements of temporal correlators. The lattice unit is determined as $a_{s}^{-1}=1.100(6)$ $\mathrm{GeV}$ from the Sommer parameter $r_{0}^{-1}=395 \mathrm{MeV}$, and thus the physical lattice volume amounts to $(2.15 \mathrm{fm})^{3} \times(4.30 \mathrm{fm})$. We use totally 1000 gauge field configurations to achieve the high statistics analysis. This is quite essential for our study, because the $5 \mathrm{Q}$ correlators for spin $3 / 2$ states are found to be rather noisy. For the quark fields, we adopt the $O(a)$-improved Wilson (clover) action on the anisotropic lattice to suppress the discretization error. We adopt four values of the hopping parameters as $\kappa=0.1210(0.0010) 0.1240$, which corresponds to $m_{\pi} / m_{\rho}=0.81,0.78,0.73$ and 
0.66 and roughly covers the region $m_{s} \lesssim m \lesssim 2 m_{s}$. By keeping $\kappa_{s}=0.1240$ fixed for s quark, we change $\kappa=0.1210-0.1240$ for $\mathrm{u}$ and $\mathrm{d}$ quarks for chiral extrapolation. In order to enhance the low-lying spectra, we use a gaussian smeared source (gaussian size of $\rho \simeq 0.4 \mathrm{fm}$ ) and point sink correlator in the Coulomb gauge. For details of the lattice QCD calculations, see Ref.[18].

\section{1 $J^{P}=3 / 2^{-} 5 \mathbf{Q}$ spectrum}

We consider 5Q spectrum in $J^{P}=3 / 2^{-}$channel in PBC. In the upper side of Figs. 1 (a), (b), (c), we show the effective mass plots for three fields, (a) the NK*-type, (b) the twisted NK*-type, (c) a diquark-type, respectively. Unless otherwise indicated, we use $\left(\kappa_{s}, \kappa\right)=(0.1240,0.1220)$ as a typical set of hopping parameters hereafter. In upper Figs. 1 (a) and (b), we find the plateaus around $25 \lesssim \tau \lesssim 35$ where the contamination from excited states as well as the backward propagation are suppressed. We perform a single-exponential fit in this region and obtain (a) $m_{5 \mathrm{Q}}=2.90$ (2) GeV, (b) $m_{5 \mathrm{Q}}=2.89(1) \mathrm{GeV}$, respectively. On the other hand, we see that the statistical error is too large in upper Fig. 1 (c), and therefore we do not use diquark-type field in this channel. A possible reason for such a large noise is that this field does not survive in the non-relativistic limit. Now, we perform the chiral extrapolation. Fig. 2 shows the 5Q masses in $J^{P}=3 / 2^{-}$channel against $m_{\pi}^{2}$. Since the data behave almost linearly in $m_{\pi}^{2}$, we adopt the linear chiral extrapolation in $m_{\pi}^{2}$. As a result, we obtain only massive $5 \mathrm{Q}$ states as (a) $m_{5 \mathrm{Q}}=2.17$ (4) $\mathrm{GeV}$, (b) $m_{5 \mathrm{Q}}=2.11$ (4) GeV, respectively, which is too heavy to be identified with $\Theta^{+}(1540)$. Note that none of 5Q states appear below the NK threshold, although this threshold is raised up by about $200-250 \mathrm{MeV}$ due to the finite extent of the spatial lattice, and the $5 \mathrm{Q}$ signal is expected to appear below the (raised) NK threshold considering the empirical mass difference between $\mathrm{N}+\mathrm{K}(1440)$ and $\Theta^{+}(1540)$.

To clarify whether our 5Q states are compact resonances or not, we perform the analysis with HBC. With the typical set of hopping parameters, it is expected that the s-wave $\mathrm{NK}^{*}$ state is raised up by $\sim 180 \mathrm{MeV}$, the d-wave NK state is lowered down by $\sim 70 \mathrm{MeV}$, and a compact 5Q state shows no difference by switching PBC to HBC. Actual lattice simulations show $m_{5 \mathrm{Q}}=2.98(1)$ $\mathrm{GeV}$ for both of $\mathrm{NK}^{*}$-type and twisted $\mathrm{NK}^{*}$-type fields, which corresponds to upper shift by 80$90 \mathrm{MeV}$. Although the shift of $m_{5 \mathrm{Q}}$ is rather small, the value of $m_{5 \mathrm{Q}}$ is consistent with the s-wave $\mathrm{NK}^{*}$ state within the statistical error. Therefore, we regard this state as an s-wave $\mathrm{NK}^{*}$ state. In this way, we do not observe any compact $5 \mathrm{Q}$ resonances in $J^{P}=3 / 2^{-}$channel below the raised s-wave $\mathrm{NK}^{*}$ threshold, i.e., in the region of $E \lesssim \sqrt{m_{N}^{2}+\vec{p}_{\text {min }}^{2}}+\sqrt{m_{K^{*}}^{2}+\vec{p}_{\text {min }}^{2}}$, with $\left|\vec{p}_{\text {min }}\right| \simeq 499 \mathrm{MeV}$.

\section{$3.2 J^{P}=3 / 2^{+} 5 \mathrm{Q}$ spectrum}

We perform the analysis for 5Q spectrum in $J^{P}=3 / 2^{+}$channel. The lower side of Figs. 1 (a), (b), (c), show the effective mass plots for (a) the $\mathrm{NK}^{*}$-type, (b) the twisted $\mathrm{NK}^{*}$-type, (c) a diquarktype, respectively. In all figures, we observe the plateaus and perform the single-exponential fit. We obtain (a) $m_{5 \mathrm{Q}}=3.34$ (3) GeV, (b) $m_{5 \mathrm{Q}}=3.11$ (4) $\mathrm{GeV}$ and (c) $m_{5 \mathrm{Q}}=3.16(2) \mathrm{GeV}$, respectively.Note that (a) agrees with the s-wave $\mathrm{N}^{*} \mathrm{~K}^{*}$ threshold while the latter two is very close to $\mathrm{NK}^{*}$ threshold. Now, we perform the chiral extrapolation. In Fig. 2, $m_{5 \mathrm{Q}}$ is plotted against $m_{\pi}^{2}$. Using the linear chiral extrapolation in $m_{\pi}^{2}$, we obtain (a) $m_{5 \mathrm{Q}}=2.64(7) \mathrm{GeV}$, (b) $m_{5 \mathrm{Q}}=2.48$ (10) $\mathrm{GeV}$ and (c) $m_{5 \mathrm{Q}}=2.42(6) \mathrm{GeV}$, respectively. Note that we observe again that all of $m_{5 \mathrm{Q}}$ data appear above the $\mathrm{NK}^{*}$ p-wave threshold, which is located above the (raised) NK p-wave threshold. 


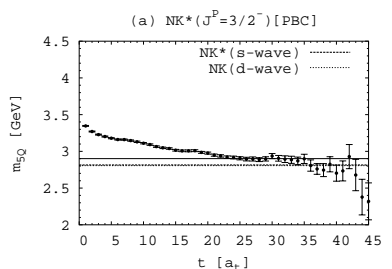

(a) $\mathrm{NK}^{*}\left(\mathrm{~J}^{\mathrm{P}}=3 / 2^{+}\right)[\mathrm{PBC}]$

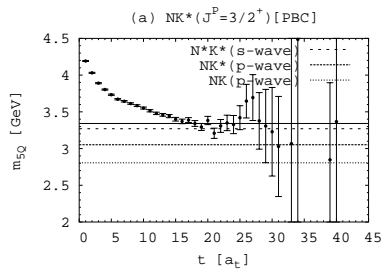

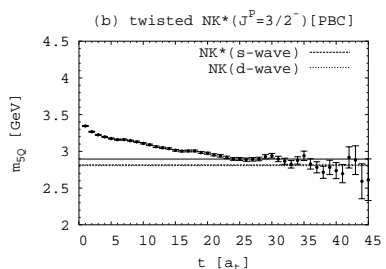

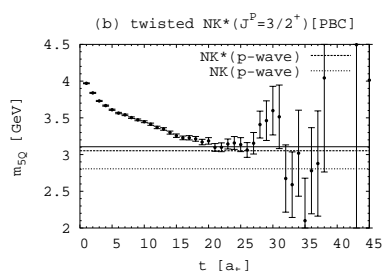

$\operatorname{diquark}\left(\mathrm{J}^{\mathrm{P}}=3 / 2^{-}\right)[\mathrm{PBC}]$
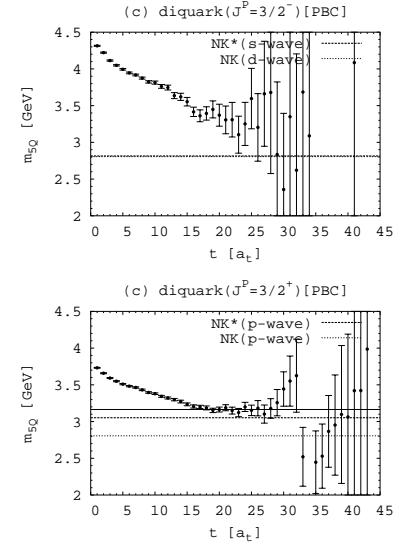

Figure 1: The 5Q effective mass plots in $J^{P}=3 / 2^{-}$(upper) and $J^{P}=3 / 2^{+}$(lower) channel in the periodic $\mathrm{BC}(\mathrm{PBC})$ for three types of fields: (a) the $\mathrm{NK}^{*}$-type, (b) the twisted $\mathrm{NK}^{*}$-type, and (c) the diquark-type. The dotted lines indicate the $\mathrm{s}(\mathrm{p})$-wave $\mathrm{NK}^{*}$ and the $\mathrm{d}(\mathrm{p})$-wave $\mathrm{NK}$ threshold for $J^{P}=3 / 2^{-}\left(3 / 2^{+}\right)$. The solid lines denote the results of the single-exponential fit performed in each plateau region.

Next, we perform the HBC analysis. For $J^{P}=3 / 2^{+}$channel, the energy shift is somewhat minor change. With the typical set of hopping parameters, it is expected that the s-wave $\mathrm{N}^{*} \mathrm{~K}^{*}$ state is raised up by $\sim 170 \mathrm{MeV}$, the p-wave $\mathrm{NK}^{*}$ state is lowered down by $\sim 60 \mathrm{MeV}$, the pwave NK state is lowered down by $\sim 70 \mathrm{MeV}$ and a compact 5Q state shows no difference by switching PBC to HBC. As a lattice result, we obtain $m_{5 \mathrm{Q}}=3.38(2) \mathrm{GeV}$ from $\mathrm{NK}^{*}$-type field. Although corresponding shift of $40 \mathrm{MeV}$ is rather small, $m_{5 \mathrm{Q}}$ is again almost consistent with the s-wave $\mathrm{N}^{*} \mathrm{~K}^{*}$ threshold. Considering its rather large statistical error, this $5 \mathrm{Q}$ state is likely to be an s-wave $\mathrm{N}^{*} \mathrm{~K}^{*}$ state. The results from other two fields are very similar to each other. We obtain (b) $m_{5 \mathrm{Q}}=3.02(3) \mathrm{GeV}$ and (c) $m_{5 \mathrm{Q}}=3.08(4) \mathrm{GeV}$, respectively. These results correspond to lower shift by $80-90 \mathrm{MeV}$, which is considered to be consistent with the $\mathrm{NK}^{*}$ p-wave behavior. Therefore, these states are likely to be an $\mathrm{NK}^{*} \mathrm{p}$-wave state. In this way, we observe no signal for compact $5 \mathrm{Q}$ resonances. Although the assignments for plateaus are still afflicted by considerable size of statistical error, we can at least state that these $5 \mathrm{Q}$ states are all massive, which locate above $\mathrm{NK}^{*}$ p-wave threshold and that these states are too heavy to be identified with $\Theta^{+}(1540)$.

\section{Summary and Conclusions}

We have studied $J^{P}=3 / 2^{ \pm}$pentaquark(5Q) baryons in anisotropic lattice QCD at the quenched level. We have employed the standard Wilson gauge action at $\beta=5.75$ on $12^{3} \times 96$ lattice with the renormalized anisotropy $a_{\mathrm{s}} / a_{\mathrm{t}}=4$. The large statistics as $N_{\text {conf }}=1000$ has played a key role in achieving a solid result in our calculation. For the quark action, we have adopted $O(a)$-improved Wilson (clover) action with the hopping parameter as $\kappa=0.1210(0.0010) 0.1240$, which roughly corresponds to $m_{s} \lesssim m \lesssim 2 m_{s}$. We have examined three types of the interpolating fields as (a) the $\mathrm{NK}^{*}$-type, (b) the twisted $\mathrm{NK}^{*}$-type, (c) the diquark-type. In $J^{P}=3 / 2^{-}$channel, we have observed plateaus in the effective mass plots except for the diquark-type field. Employing the linear chiral extrapolations in $m_{\pi}^{2}$, we have obtained $m_{5 \mathrm{Q}} \simeq 2.17$ and $2.11 \mathrm{GeV}$ in the chiral limit for the $\mathrm{NK}^{*}$-type and the twisted $\mathrm{NK}^{*}$-type correlators, respectively. We have performed the HBC analysis and have found that both of the observed $5 \mathrm{Q}$ states are s-wave $\mathrm{NK}^{*}$ scattering states. In $J^{P}=3 / 2^{+}$ channel, we have recognized plateaus in all the three effective mass plots. The chiral extrapolations 

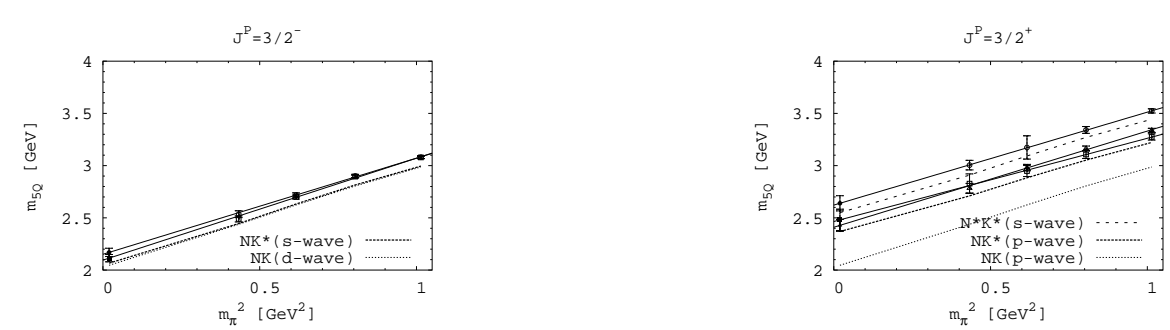

Figure 2: $m_{5 \mathrm{Q}}$ in $J^{P}=3 / 2^{-}$(left) and $3 / 2^{+}$(right) channel against $m_{\pi}^{2}$ for the three fields, i.e., (circle) the $\mathrm{NK}^{*}$-type, (box) the twisted $\mathrm{NK}^{*}$-type, and (triangle) a diquark-type. Data from diquark-type are not plotted in $J^{P}=3 / 2^{-}$because a plateau is not obtained in the effective mass plot. Open symbols denote the direct lattice results, while the closed symbols and the solid lines represent the results of the chiral extrapolations. have lead to $m_{5 \mathrm{Q}} \simeq 2.64,2.48,2.42 \mathrm{GeV}$ for the $\mathrm{NK}^{*}$-type, twisted $\mathrm{NK}^{*}$-type and the diquark-type correlator, respectively. HBC analyses have been performed and the observed 5Q states in both of the twisted $\mathrm{NK}^{*}$-type and the diquark-type correlators are most likely to be $\mathrm{NK}^{*}$ p-wave states. For the $\mathrm{NK}^{*}$-type field, it is most likely to be an s-wave $\mathrm{N}^{*} \mathrm{~K}^{*}$ state, although more statistics is needed to draw a definite conclusion. At any rate, whatever the real nature of these 5Q states may be, they are all considerably massive states in the physical quark mass region in both of $J^{P}=3 / 2^{ \pm}$ channels, and cannot be identified as $\Theta^{+}(1540)$ without involving a significantly large chiral effect. For further studies, it is important to perform the systematic studies of the 5Q states using such as (1) unquenched full lattice QCD, (2) finer and larger volume lattice, (3) chiral fermion with small mass, (4) more sophisticated interpolating field reflecting the structure of 5Q, etc.

\section{References}

[1] LEPS Collaboration, T. Nakano et al., Phys. Rev. Lett. 91, 012002 (2003).

[2] For a review of the experimental status, K.H. Hicks, hep-ex/0504027, and references therein.

[3] For a review, M. Oka, Prog. Theor. Phys. 112, 1 (2004), and references therein.

[4] A. Hosaka, Phys. Lett. B571, 55 (2003).

[5] R.L. Jaffe and F. Wilczek, Phys. Rev. Lett. 91, 232003 (2003).

[6] F. Csikor, Z. Fodor, S.D. Katz, and T.G. Kovacs, JHEP 0311, 070 (2003).

[7] S. Sasaki, Phys. Rev. Lett. 93, 152001 (2004).

[8] T.W. Chiu and T.H. Hsieh, hep-ph/0403020.

[9] N. Mathur et al., Phys. Rev. D70, 074508 (2004).

[10] N. Ishii, T. Doi, H. Iida, M. Oka, F. Okiharu, and H. Suganuma, Phys. Rev. D71, 034001 (2005).

[11] T.T. Takahashi, T. Umeda, T. Onogi and T. Kunihiro, Phys. Rev. D71, 114509 (2005).

[12] B.G. Lasscock et al., Phys. Rev. D72, 014502 (2005).

[13] C. Alexandrou and A. Tsapalis, hep-lat/0503013.

[14] F. Csikor, Z. Fodor, S.D. Katz, T.G. Kovács, and B.C. Tóth, hep-lat/0503012.

[15] K. Holland, and K.J. Juge, hep-lat/0504007.

[16] B.G. Lasscock et al., hep-lat/0504015.

[17] J. Sugiyama, T. Doi, and M. Oka, Phys. Lett. B 581, 167 (2004).

[18] N. Ishii, T. Doi, Y. Nemoto, M. Oka and H. Suganuma, hep-lat/0506022, Phys. Rev. D72 (2005). 